\title{
Unoccupied topological states on bismuth chalcogenides
}

\author{
D. Niesner, ${ }^{1}$ Th. Fauster, ${ }^{1, *}$ S. V. Eremeev, ${ }^{2,3}$ T. V. Menshchikova, ${ }^{3}$ Yu. M. Koroteev, ${ }^{2,3}$ A. P. Protogenov, ${ }^{4,5}$ E. V. Chulkov, ${ }^{5,6}$ \\ O. E. Tereshchenko, ${ }^{7}$ K. A. Kokh, ${ }^{8}$ O. Alekperov, ${ }^{9}$ A. Nadjafov, ${ }^{9}$ and N. Mamedov ${ }^{9}$ \\ ${ }^{1}$ Lehrstuhl für Festkörperphysik, Universität Erlangen-Nürnberg, D-91058 Erlangen, Germany \\ ${ }^{2}$ Institute of Strength Physics and Materials Science, 634021 Tomsk, Russia \\ ${ }^{3}$ Tomsk State University, 634050 Tomsk, Russia \\ ${ }^{4}$ Institute of Applied Physics, 603950 Nizhny Novgorod, Russia \\ ${ }^{5}$ Donostia International Physics Center (DIPC), 20018 San Sebastián/Donostia, Basque Country, Spain \\ ${ }^{6}$ Departamento de Física de Materiales UPV/EHU and Centro de Física de Materiales CFM and Centro Mixto CSIC-UPV/EHU, \\ 20080 San Sebastián/Donostia, Basque Country, Spain \\ ${ }^{7}$ Institute of Semiconductor Physics, 630090 Novosibirsk, Russia \\ ${ }^{8}$ Institute of Geology and Mineralogy, 630090 Novosibirsk, Russia \\ ${ }^{9}$ Institute of Physics, Azerbaijan National Academy of Sciences, AZ1143 Baku, Azerbaijan
}

(Received 16 May 2012; revised manuscript received 18 October 2012; published 5 November 2012)

\begin{abstract}
The unoccupied part of the band structure of topological insulators $\mathrm{Bi}_{2} \mathrm{Te}_{x} \mathrm{Se}_{3-x}(x=0,2,3)$ is studied by angle-resolved two-photon photoemission and density functional theory. For all surfaces linearly dispersing surface states are found at the center of the surface Brillouin zone at energies about $1.3 \mathrm{eV}$ above the Fermi level. Theoretical analysis shows that this feature appears in a spin-orbit-interaction-induced and inverted local energy gap. This inversion is insensitive to variation of electronic and structural parameters in $\mathrm{Bi}_{2} \mathrm{Se}_{3}$ and $\mathrm{Bi}_{2} \mathrm{Te}_{2} \mathrm{Se}$. In $\mathrm{Bi}_{2} \mathrm{Te}_{3}$ small structural variations can change the character of the local energy gap, depending on whether or not an unoccupied Dirac state exists. Circular dichroism measurements confirm the expected spin texture. From these findings we assign the observed state to an unoccupied topological surface state.
\end{abstract}

DOI: 10.1103/PhysRevB.86.205403

PACS number(s): 73.20.-r, 79.60.Bm

\section{INTRODUCTION}

Three-dimensional topological insulators (TIs) are insulators in bulk and metals at the surface. The metallic character of a TI surface is determined by a massless Dirac state that crosses the Fermi level $E_{\mathrm{F}}{ }^{1-3}$ This spin-polarized linearly dispersing surface state arises from a symmetry inversion of the bulk bands at band gap edges owing to strong spinorbit interaction (SOI). Various new phenomena in TIs were predicted, like dissipationless spin transport, ${ }^{4}$ formation of Majorana fermions in the presence of superconductors, ${ }^{5}$ and magnetic monopoles. ${ }^{6}$

Most of these phenomena deal with low-energy electronic excitations and transport. Little experimental work has been performed on excited electronic states and their dynamics. The best studied three-dimensional TIs at present are bismuth chalcogenides. The equilibrium band structure of these TIs is well understood ${ }^{3,7-13}$ and the spin texture of the topological surface state (TSS) was observed both indirectly by use of circular dichroism $(\mathrm{CD})^{14}$ and directly by spin-resolved experiments. $^{8,15}$

The materials are usually intrinsically $n$-doped, which complicates direct spectroscopic access to excited electrons in a TSS. In addition to an earlier inverse photoemission investigation, ${ }^{16}$ a recent study was performed by Sobota et al. demonstrating that a persistent occupation of the Dirac cone close to the Fermi level in $p$-doped $\mathrm{Bi}_{2} \mathrm{Se}_{3}$ can be supported even picoseconds after an initial optical excitation. ${ }^{17}$ The transient carrier population on $\mathrm{Bi}_{2} \mathrm{Te}_{3}$ has also been reported. ${ }^{18}$ The cooling of Dirac fermions was studied on intrinsic ( $n$-doped) $\mathrm{Bi}_{2} \mathrm{Se}_{3}$. ${ }^{19}$

Here we present a different approach by investigating higher-excited TSSs in $\mathrm{Bi}_{2} \mathrm{Te}_{x} \mathrm{Se}_{3-x}(x=0,2,3)$. In the calculated band structures symmetry-inverted band gaps are identified which support an empty Dirac cone. Experimental proof is given by monochromatic two-photon photoemission (2PPE). Calculations together with CD measurements reveal the characteristic spin structure of a TSS.

\section{METHODS}

\section{A. Experiment}

Angle-resolved monochromatic 2PPE and one-photon photoemission (ARPES) experiments were conducted using the third and fourth harmonic (4.65 and 6.2-eV photon energy) of a titanium:sapphire oscillator with a repetition rate of $80 \mathrm{MHz}$ and pulse lengths of about $100 \mathrm{fs}^{20,21}$ In both cases the beam is initially $p$-polarized with an incidence angle of $45^{\circ}$. Circular polarization of the third harmonic necessary for $\mathrm{CD}$ experiments was obtained using a $\lambda / 4$ waveplate. Two-dimensional momentum distribution patterns (MDCs) at a constant kinetic energy were recorded using an ellipsoidal "display-type" analyzer at an energy and angular resolution of $50 \mathrm{meV}$ and $3^{\circ}$, respectively. ${ }^{21,22}$ Angle-resolved spectra for the intensity maps were acquired by a hemispherical analyzer with a resolution of $34 \mathrm{meV}$ and $1.6^{\circ}$, respectively. ${ }^{20}$

$\mathrm{Bi}_{2} \mathrm{Te}_{2} \mathrm{Se}$ was prepared from presynthesized mixtures of $\mathrm{Bi}_{2} \mathrm{Te}_{3}$ and $\mathrm{Bi}_{2} \mathrm{Se}_{3}$, which, in turn, were prepared from elementary $\mathrm{Bi}, \mathrm{Te}$, and $\mathrm{Se}$ of $99.999 \%$ purity. Crystal growth for all three compounds was done in sealed quartz ampoules coated with a carbon layer. For recrystallization we used a vertical variant of the modified Bridgman method. ${ }^{23}$ The resulting ingots consisted of one or several large singlecrystalline blocks. $\mathrm{Bi}_{2} \mathrm{Se}_{3}, \mathrm{Bi}_{2} \mathrm{Te}_{3}$, and $\mathrm{Bi}_{2} \mathrm{Te}_{2}$ Se samples were naturally $n$-doped (through the formation of defects) with 
a carrier concentration in the range of $(1-9) \times 10^{18} \mathrm{~cm}^{-3}$. Samples were cleaved in vacuum at a pressure of $<5 \times 10^{-6} \mathrm{~Pa}$ and then transferred to ultrahigh vacuum (pressure, $<1 \times$ $10^{-8} \mathrm{~Pa}$ ), where they were cooled to $90 \mathrm{~K}$ for measurements. Sample quality and orientation were checked by low-energy electron diffraction (LEED) showing a sharp threefold pattern. According to LEED patterns the samples were oriented with the laser beams incident parallel to the $\overline{\Gamma M}$ mirror plane or along the $\overline{\Gamma K}$ direction.

\section{B. Theory}

Because the description of the unoccupied states is a delicate issue of density functional theory (DFT), we employ two different computer codes that are based on DFT. The main part of the electronic structure calculations was performed within the density functional formalism implemented in VASP. ${ }^{24,25} \mathrm{We}$ used the all-electron projector augmented-wave (PAW ${ }^{26,27}$ basis sets with the generalized gradient approximation of Perdew, Burke, and Ernzerhof (PBE) ${ }^{28}$ to the exchange-correlation potential. For bulk band structure calculations the local density approximation (LDA) ${ }^{29}$ to the exchange-correlation potential was also tested. The experimental lattice parameters were used for calculation of all the considered compounds while atom positions within the unit cell were optimized. The Hamiltonian contains scalar relativistic corrections, and the SOI is taken into account by the second variation method. ${ }^{30}$ To simulate the semi-infinite $\mathrm{Bi}_{2} \mathrm{Te}_{x} \mathrm{Se}_{3-x}(111)$ we use a slab composed of nine quintuple layers (QLs).

The second approach used for electronic structure calculations is the full-potential linearized augmented planewave (FLAPW) method as implemented in the FLEUR code ${ }^{31}$ with PBE for the exchange-correlation potential. Spin-orbit coupling was included in the self-consistent calculations as described in Ref. 32. The FLAPW basis has been extended by conventional local orbitals to treat quite shallow semicore $d$ states. Additionally, to describe high-lying unoccupied states accurately, we have included for each atom one local orbital per angular momentum up to $l=3$. The fundamental quantities are consistently reproduced by both methods ${ }^{33}$ except for the case $\mathrm{Bi}_{2} \mathrm{Te}_{3}$ which is addressed below in detail.

To study the effect of dispersion interactions we use the van der Waals nonlocal correlation functional (vdW-DF) as implemented in the VASP code. ${ }^{34}$ Both lattice parameters and internal atomic positions were optimized in this approach.

\section{RESULTS}

\section{A. Unoccupied topological state}

We start with the ternary tetradymite compound $\mathrm{Bi}_{2} \mathrm{Te}_{2} \mathrm{Se}$, which recently was shown to be a three-dimensional TI by ARPES measurements. ${ }^{35,36}$ The crystal structure of this compound is obtained by replacing the central Te layer of each $\mathrm{QL}$ of $\mathrm{Bi}_{2} \mathrm{Te}_{3}$ with a Se layer. The results of photoemission experiments and DFT calculations on $\mathrm{Bi}_{2} \mathrm{Te}_{2} \mathrm{Se}$ are shown in Fig. 1. Shaded regions in the calculated band structure depict projected bulk bands, while filled (red) circles indicate increased localization in the first $\mathrm{Bi}_{2} \mathrm{Te}_{2} \mathrm{Se}$ QL. From the ARPES data [Fig. 1(a)] the Dirac cone in the occupied part of the band structure is found $0.25 \mathrm{eV}$ below the $E_{\mathrm{F}}$. Depending
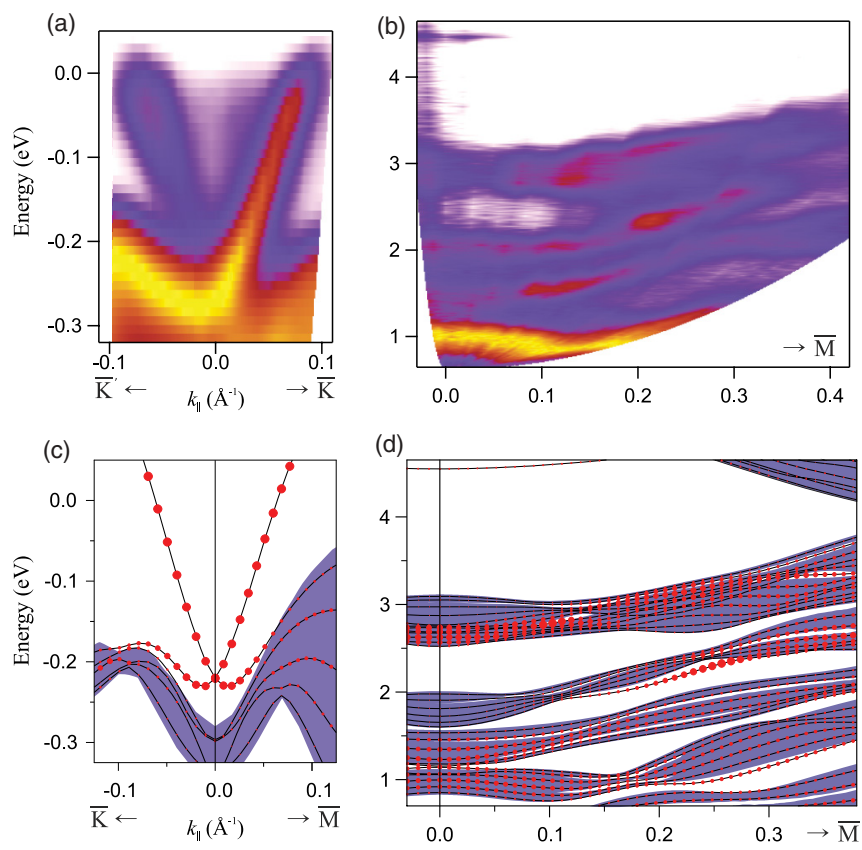

FIG. 1. (Color online) (a, b) Measured and (c, d) calculated data on the band structure of $\mathrm{Bi}_{2} \mathrm{Te}_{2} \mathrm{Se}$. (a, c) Occupied states; (b, d) unoccupied states. In (c) and (d) shaded regions indicate projected bulk bands, whereas filled (red) circles indicate increased localization within the topmost QL.

on the crystal growth conditions values between 0.1 and $0.4 \mathrm{eV}$ have been found. ${ }^{35,36}$ In the 2PPE data [Fig. 1(b)] all prominent features can be attributed to unoccupied bands in the calculations [Fig. 1(d)] and we conclude that the 2PPE process is dominated by intermediate states. The measured bands of high intensity coincide well with surface resonances in the calculation, giving evidence of a high surface sensitivity. A similar degree of agreement between theory and experiment is also found for $\mathrm{Bi}_{2} \mathrm{Se}_{3}$ and $\mathrm{Bi}_{2} \mathrm{Te}_{3}$ surfaces. The present results are in qualitative agreement with the inverse photoemission spectra, which show three conduction band peaks. ${ }^{16}$

In the following we focus on the projected bulk band gaps at energies above the $E_{\mathrm{F}}$. Figures 2(a) and 2(d) show a closeup of such a gap for $\mathrm{Bi}_{2} \mathrm{Te}_{2} \mathrm{Se}$. In fact both 2PPE and DFT data show that it is bridged by two linearly dispersing bands which cross at the $\bar{\Gamma}$ point. At this energy it appears in MDCs as a single spot evolving into a circle towards higher energies [see also Fig. 4(a) and Ref. 37]. At energies below the crossing point it turns into a surface resonance degenerate with bulk bands.

\section{B. Theoretical considerations}

The appearance of the Dirac state in the conduction-band local gap raises the question: Is this surface state topologically protected? In contrast to the Dirac state located in the principal energy gap, the new massless Dirac state is located in a local energy gap, and upon going along a path between time-reversal invariant momenta in the Brillouin zone the gap in the spectrum of bulk states closes and opens.

The origin of the reduction of the contribution made by extended states can be found by comparing the continuous and lattice versions of the $\mathbb{Z}_{2}$ invariant. Analysis of the relation ${ }^{38}$ 

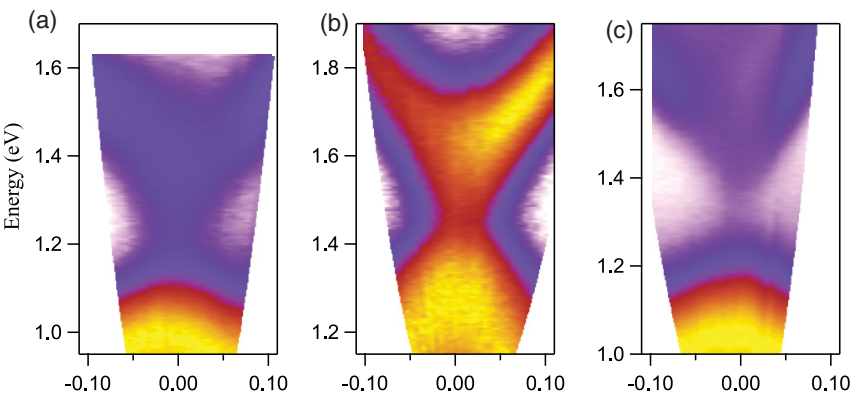

$\mathrm{Bi}_{2} \mathrm{Te}_{2} \mathrm{Se}$
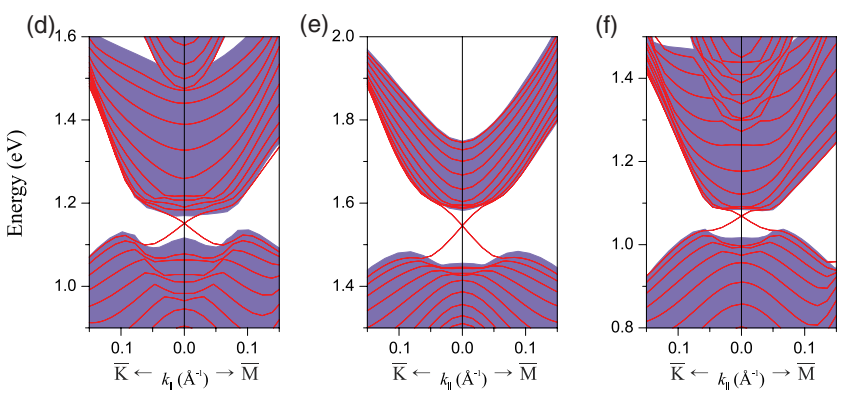

FIG. 2. (Color online) Closeup of the band gap under investigation for the three materials. No azimuthal dependence was observed close to $\bar{\Gamma}$ and the sample orientation was optimized to access the Dirac point in experiments.

$(-1)^{\nu_{0}}=(-1)^{2 P_{3}}$ of the index ${ }^{1} v_{0}$ in the theory of TIs with the winding number $2 P_{3}$ does not solve the problem. It is shown in Ref. 39 that the $\mathbb{Z}_{2}$ invariant in the continuous case is alternatively expressed as

$$
D=\frac{1}{2 \pi i}\left[\oint_{\partial \mathcal{B}^{-}} A-\int_{\mathcal{B}^{-}} F\right] \bmod 2,
$$

where $\mathcal{B}^{-}=[-\pi, \pi] \otimes[-\pi, 0]$ is half of the Brillouin zone, $A=\operatorname{Tr} \psi^{\dagger} d \psi$ and $F=d A$ are the Berry gauge potential and the associated field strength, respectively, and $\psi(k)$ is the $2 M(k)$-dimensional ground-state multiplet. The lattice analog of Eq. (1) is ${ }^{40}$

$$
\begin{aligned}
D_{\mathrm{L}} & \equiv \frac{1}{2 \pi i}\left[\sum_{k \in \partial \mathcal{B}^{-}} A(k)-\sum_{k \in \mathcal{B}^{-}} F(k)\right] \\
& =-\sum_{k \in \mathcal{B}^{-}} n(k) \bmod 2,
\end{aligned}
$$

since $\sum_{k \in \mathcal{B}^{-}} F(k)=\sum_{k \in \partial \mathcal{B}^{-}} A(k)+2 \pi i \sum_{k \in \mathcal{B}^{-}} n(k) . n(k)$ in this equation are integers and $n(k) \bmod 2 \in \mathbb{Z}_{2}$ due to the residual $U(1)$ invariance. $^{40}$

From Eq. (2) we infer that the reason for cancellation of the contribution made by bulk spectrum states is the compactness of the lattice gauge theory. The existence of the second Dirac cone points out the nontrivial value $D_{\mathrm{L}}=1 \bmod 2$ of the $\mathbb{Z}_{2}$ invariant in this topologically protected state.

Let us consider now the bulk conduction band of $\mathrm{Bi}_{2} \mathrm{Te}_{2} \mathrm{Se}$ in the energy range of interest. Without spin-orbit coupling the second and third conduction bands are degenerate along the $\Gamma \mathrm{Z}$ direction [Fig. 3(a)]. SOI lifts this degeneracy and opens a gap between these bands [Fig. 3(b)]. Thus, the gap supporting the new X-shaped surface state in the conduction band originates from SOI. In contrast to the principal $\Gamma$-gap
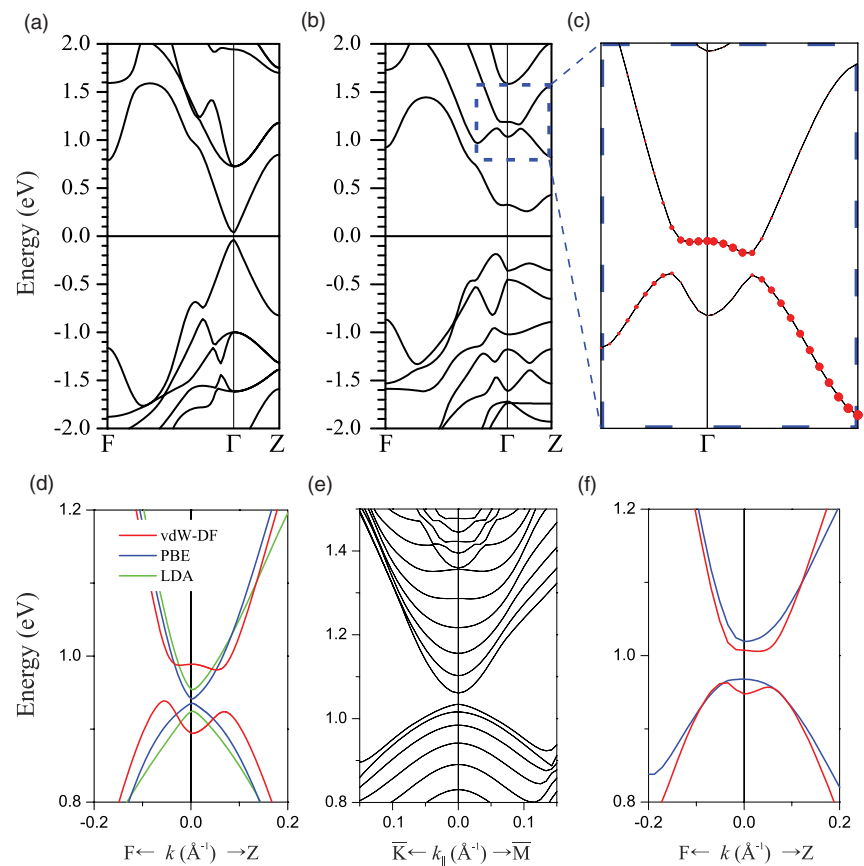

FIG. 3. (Color online) Bulk band structure of $\mathrm{Bi}_{2} \mathrm{Te}_{2} \mathrm{Se}$ calculated with VASP (only $F-\Gamma$ and $\Gamma-Z$ directions are shown): (a) without and (b) with SOI included. (c) Magnified view of the dashed (blue) square in (b) with the weight of Se states marked in the second and third conduction bands near the $\Gamma$ point. (d) Bulk spectrum of $\mathrm{Bi}_{2} \mathrm{Te}_{3}$ in the vicinity of the local gap within the vdW-DF, PBE, and LDA approaches. (e) Nine-QL-slab electronic structure of $\mathrm{Bi}_{2} \mathrm{Te}_{3}$ within the PBE. (f) Bulk spectrum of $\mathrm{Bi}_{2} \mathrm{Te}_{3}$ as calculated by the FLEUR code with different experimental parameters: upper (blue) line in each pair, Ref. 41; lower (red) line in each pair, Ref. 42.

edges, which are composed of inverted $\mathrm{Bi}$ and $\mathrm{Te}$ states, both the upper and the lower bands of the conduction band $\Gamma$ gap are mostly composed of $\mathrm{Bi}$ states, however, the $\mathrm{Se}$ states contribute to these bands as well. As one can see in Fig. 3(c) in the vicinity of $\Gamma$ there are two parabolic-like bands, gapped at points, where they are inverted due to the central $\mathrm{Se}$ atom contribution. This inversion of the local $\Gamma$-gap edges in the conduction band along with the presence at the same energy of the gaps at $\mathrm{F}$ and $\mathrm{L}$ time-reversal invariant momenta of the bulk Brillouin zone (which are projected onto the $\bar{M}$ point of the two-dimensional Brillouin zone) is responsible for the emergence of the unoccupied Dirac-like surface state at $\mathrm{Bi}_{2} \mathrm{Te}_{2} \mathrm{Se}(111)$.

As one can see in Figs. 2(e) and 2(f), the FLEUR results show a topological conduction-band surface state arising in $\mathrm{Bi}_{2} \mathrm{Se}_{3}$ and $\mathrm{Bi}_{2} \mathrm{Te}_{3}$, which is fully consistent with the outcome of the experiment. The VASP vdW-DF results are almost the same: they show a 10-20 meV smaller unoccupied gap and a slightly different position of the surface state band crossing within this gap. The arising of an unoccupied cone in $\mathrm{Bi}_{2} \mathrm{Se}_{3}$ and $\mathrm{Bi}_{2} \mathrm{Te}_{3}$, as in $\mathrm{Bi}_{2} \mathrm{Te}_{2} \mathrm{Se}$, results from inversion of the $\mathrm{QL}$ central atom states of the SOI-induced $\Gamma$ local gap. The LDA and $\mathrm{PBE}$ calculations performed without taking into account the vdW forces for $\mathrm{Bi}_{2} \mathrm{Te}_{2} \mathrm{Se}$ and $\mathrm{Bi}_{2} \mathrm{Se}_{3}$ give the inverted gap too, while in the case of $\mathrm{Bi}_{2} \mathrm{Te}_{3}$ the SOI-induced gap is not inverted ${ }^{33}$ [Fig. 3(d)]. The dissimilar character of the 
gap in $\mathrm{Bi}_{2} \mathrm{Te}_{3}$ in $\mathrm{PBE}$ and $\mathrm{LDA}$ versus vdW-DF results in the absence of the unoccupied cone in the surface band structure [Fig. 3(e)]. As mentioned above the different calculations were accompanied by optimization of the crystal structure. Thus, vdW-DF optimization for all systems under consideration changes the $a$ and $c$ lattice parameters in the range of $0.5 \%-1 \%$ and $0.1 \%-2 \%$ compared to experimental values, respectively. At the same time both the full structural optimization and the relaxation of atomic positions do not change the fractional atomic coordinates more than $10^{-3}$. To determine which factor (exchange-correlation approximation or structural parameters) is more important for the change in the gap character, we performed calculations of the bulk electronic structure of $\mathrm{Bi}_{2} \mathrm{Te}_{3}$ with two slightly different experimental parameters ${ }^{41,42}$ with the same exchange-correlation approximation. As one can see in Fig. 3(f) this small variation in the crystal structure changes the character of the gap. The latter means that the

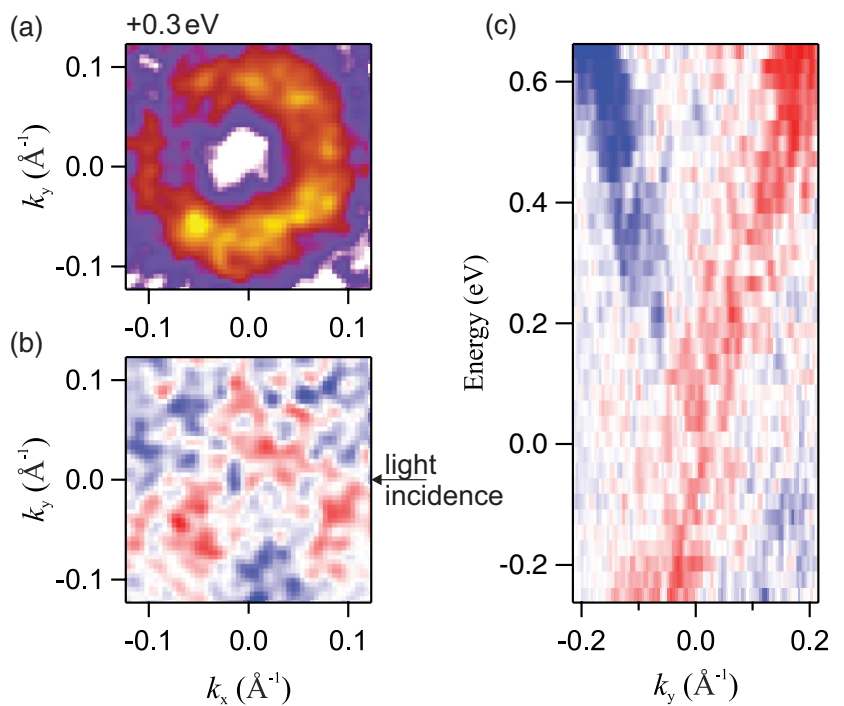

(d)
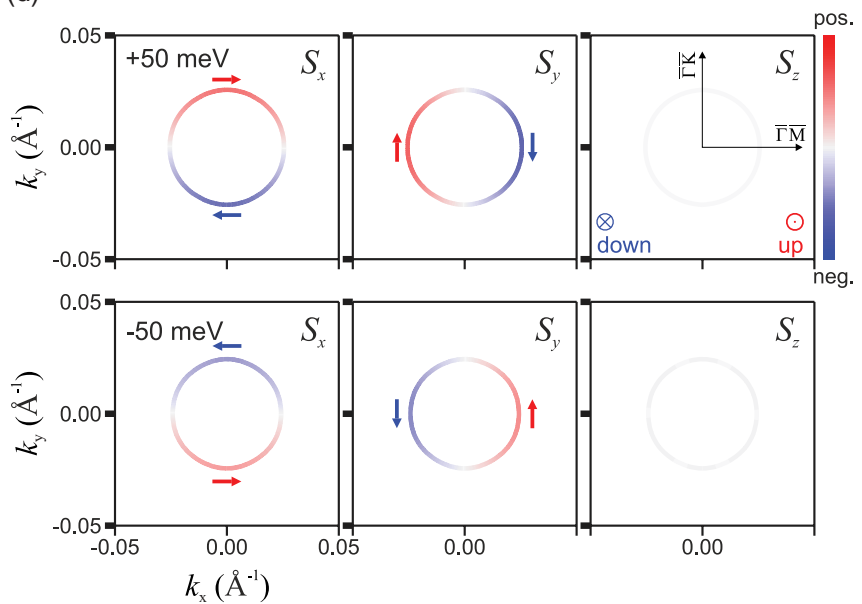

FIG. 4. (Color online) MDCs of (a) the sum and (b) the difference of the intensities for left- and right-polarized light for an energy of $300 \mathrm{meV}$ above the Dirac point for $\mathrm{Bi}_{2} \mathrm{Se}_{3}$. (c) Circular dichroism of unoccupied Dirac cone along the $\overline{\Gamma K}$ direction. (d) Calculated spin structure of the unoccupied cone as represented by spin projections $S_{x}, S_{y}$, and $S_{z}$ at an energy of $\pm 50 \mathrm{meV}$ with respect to the crossing point. emergence of the unoccupied Dirac state in $\mathrm{Bi}_{2} \mathrm{Te}_{3}$ could be sensitive to the sample preparation. As shown in Ref. 43 even a small deviation from a stoichiometric composition in $\mathrm{Bi}_{2} \mathrm{Te}_{3}$ results in a change in the lattice parameters. The data measured in the present experiment demonstrate a relatively weak intensity in the cone region [Fig. 2(c)], which may be related to the delicate stability of the unoccupied TSS in the $\mathrm{Bi}_{2} \mathrm{Te}_{3}$ sample used. On the other hand, the highest intensity observed for $\mathrm{Bi}_{2} \mathrm{Se}_{3}$ [Fig. 2(b)] reflects the more localized character of the TSS, which is a consequence of the wider gap in $\mathrm{Bi}_{2} \mathrm{Se}_{3}$ compared to other materials.

\section{Circular dichroism}

Additional evidence of the topological character of the unoccupied surface state emerges from its spin structure, which can be accessed experimentally by the use of $\mathrm{CD}$. Therefore MDCs were recorded using left- and right-handed circularly polarized light incident in the $k_{x}-k_{z}$ plane in Figs. 4(a) and 4(b). The sum $I_{\sigma+}+I_{\sigma-}$ and the difference $I_{\sigma+}-I_{\sigma-}$ of the intensities are shown for $\mathrm{Bi}_{2} \mathrm{Se}_{3}$ at an energy $300 \mathrm{meV}$ above the Dirac point. The normalized asymmetry covers a range of $\pm 12 \%$, somewhat smaller than that observed by ARPES for the occupied Dirac cone, where values of $30 \%$ for $\mathrm{Bi}_{2} \mathrm{Se}_{3}{ }^{44}$ and up to $40 \%$ for $\mathrm{Bi}_{2} \mathrm{Te}_{3}{ }^{45,46}$ have been reported. The lower asymmetry in our experiment might be attributed to the lower angular and energy resolution than in the ARPES experiments, leading to an increased unpolarized background in the narrow band gap. The two transitions in 2PPE might also have opposite contributions to the $\mathrm{CD}$ pattern, reducing the observed asymmetry. The MDCs show the expected ring and the CD pattern shows the threefold symmetry of the substrate as observed farther away from the Dirac point for the occupied TSS. ${ }^{14,45,47}$ Figure 4(c) demonstrates the development of the CD pattern over a wide energy range along the $\bar{\Gamma} \mathrm{K}$ direction. The dichroism is antisymmetric, indicating an opposite spin orientation for the two branches and a change of sign at the crossing point.

Figure 4(d) presents the calculated orientation of the electron spin within the surface state. The calculated spin-resolved constant energy contours below and above the crossing point of the unoccupied cone state show an ideal circular shape of the constant energy contours, in agreement with the present experiment, and in-plane spin polarization (the out-of-plane component $S_{z}$ is negligibly small) with a positive (clockwise) spin helicity in the upper part and a negative helicity in the lower part of the cone, i.e., the same helicity as in the Dirac cone in the principal energy gap. Note that these calculations could not be performed for the energy of Fig. 4(b), which exhibits threefold symmetry. The degeneracy with bulk bands prohibits a reliable extraction of the spin structure in this region.

\section{SUMMARY AND CONCLUSIONS}

In summary, we presented a study of the unoccupied part of the band structure of bismuth chalcogenides. Empty Dirac cones on $\mathrm{Bi}_{2} \mathrm{Se}_{3}, \mathrm{Bi}_{2} \mathrm{Te}_{2} \mathrm{Se}$, and $\mathrm{Bi}_{2} \mathrm{Te}_{3}$ are identified in both DFT calculations and 2PPE experiments by their linear dispersion and helical spin structure. Their existence depends on a symmetry-inverted band gap. In the case of 
$\mathrm{Bi}_{2} \mathrm{Te}_{3}$ this inversion is sensitive to small variations in the structural parameters, which can cause dependence of the empty Dirac cone in this system on sample growth conditions. The origin of the reduction in the bulk non-time-reversalinvariant momentum state contribution has been found using the $\mathbb{Z}_{2}$ invariant. The observed unoccupied TSS opens a new route to measurements on excitations in and between topological states and has implications for the interpretation of the observed polarization-dependent photocurrents. ${ }^{48}$ For TI-semiconductor junctions the unoccupied TSS might pave a way to inject and control spin-polarized currents in the semiconductor conduction band. The image-potential state seen at an energy of $4.5 \mathrm{eV}$ in Fig. 1(b) corresponds to the situation discussed for magnetic monopoles. ${ }^{6}$ Time-resolved photoemission experiments on image-potential states and TSSs are in progress.

\section{ACKNOWLEDGMENTS}

We thank Philip Hofmann for supplying additional topological insulator samples showing similar results. S.V.E. thanks Dirk Lamoen and I. A. Nechaev for fruitful discussions. E.V.C. acknowledges partial support from the Basque Country Government, Departamento de Educación, Universidades e Investigación (Grant No. IT-366-07), and the Spanish Ministerio de Ciencia e Innovación (Grant No. FIS2010-19609-C02-00).
*Correspondence author. fauster@physik.uni-erlangen.de

${ }^{1}$ L. Fu, C. L. Kane, and E. J. Mele, Phys. Rev. Lett. 98, 106803 (2007).

${ }^{2}$ X.-L. Qi, T. L. Hughes, and S.-C. Zhang, Phys. Rev. B 78, 195424 (2008).

${ }^{3}$ H. Zhang, C.-X. Liu, X.-L. Qi, X. Dai, Z. Fang, and S.-C. Zhang, Nature Phys. 5, 438 (2009).

${ }^{4}$ P. Roushan, J. Seo, C. V. Parker, Y. S. Hor, D. Hsieh, D. Qian, A. Richardella, M. Z. Hasan, R. J. Cava, and A. Yazdani, Nature 460, 1106 (2009).

${ }^{5}$ L. Fu and C. L. Kane, Phys. Rev. Lett. 100, 096407 (2008).

${ }^{6}$ X.-L. Qi, R. Li, J. Zang, and S.-C. Zhang, Science 323, 1184 (2009).

${ }^{7}$ Y. L. Chen, J. G. Analytis, J.-H. Chu, Z. K. Liu, S.-K. Mo, X. L. Qi, H. J. Zhang, D. H. Lu, X. Dai, Z. Fang, S. C. Zhang, I. R. Fisher, Z. Hussain, and Z.-X. Shen, Science 325, 178 (2009).

${ }^{8}$ D. Hsieh, Y. Xia, D. Qian, L. Wray, J. H. Dil, F. Meier, J. Osterwalder, L. Patthey, J. G. Checkelsky, N. P. Ong, A. V. Fedorov, H. Lin, A. Bansil, D. Grauer, Y. S. Hor, R. J. Cava, and M. Z. Hasan, Nature 460, 1101 (2009).

${ }^{9}$ Y. Xia, D. Qian, D. Hsieh, L. Wray, A. Pal, H. Lin, A. Bansil, D. Grauer, Y. S. Hor, R. J. Cava, and M. Z. Hasan, Nature Phys. 5, 398 (2009).

${ }^{10}$ O. V. Yazyev, J. E. Moore, and S. G. Louie, Phys. Rev. Lett. 105, 266806 (2010).

${ }^{11}$ S. V. Eremeev, Y. M. Koroteev, and E. V. Chulkov, JETP Lett. 91, 387 (2010).

${ }^{12}$ K. Kuroda, M. Arita, K. Miyamoto, M. Ye, J. Jiang, A. Kimura, E. E. Krasovskii, E. V. Chulkov, H. Iwasawa, T. Okuda, K. Shimada, Y. Ueda, H. Namatame, and M. Taniguchi, Phys. Rev. Lett. 105, 076802 (2010).

${ }^{13}$ M. Bianchi, D. Guan, S. Bao, J. Mi, B. Iversen, P. King, and P. Hofmann, Nature Commun. 1, 128 (2010).

${ }^{14}$ Y. H. Wang, D. Hsieh, D. Pilon, L. Fu, D. R. Gardner, Y. S. Lee, and N. Gedik, Phys. Rev. Lett. 107, 207602 (2011).

${ }^{15}$ S. V. Eremeev, G. Landolt, T. V. Menshchikova, B. Slomski, Y. M. Koroteev, Z. S. Aliev, M. B. Babanly, J. Henk, A. Ernst, L. Patthey, A. Eich, A. A. Khajetoorians, J. Hagemeister, O. Pietzsch, J. Wiebe, R. Wiesendanger, P. M. Echenique, S. S. Tsirkin, I. R. Amiraslanov, J. H. Dil, and E. V. Chulkov, Nature Commun. 3, 635 (2012)

${ }^{16}$ Y. Ueda, A. Furuta, H. Okuda, M. Nakatake, H. Sato, H. Namatame, and M. Taniguchi, J. Electron Spectrosc. Relat. Phenom. 101, 677 (1999).
${ }^{17}$ J. A. Sobota, S. Yang, J. G. Analytis, Y. L. Chen, I. R. Fisher, P. S. Kirchmann, and Z.-X. Shen, Phys. Rev. Lett. 108, 117403 (2012).

${ }^{18}$ M. Hajlaoui, E. Papalazarou, J. Mauchain, G. Lantz, N. Moisan, D. Boschetto, Z. Jiang, I. Miotkowski, Y. P. Chen, A. TalebIbrahimi, L. Perfetti, and M. Marsi, Nano Lett. 12, 3532 (2012).

${ }^{19}$ Y. H. Wang, D. Hsieh, E. J. Sie, H. Steinberg, D. R. Gardner, Y. S. Lee, P. Jarillo-Herrero, and N. Gedik, Phys. Rev. Lett. 109, 127401 (2012).

${ }^{20}$ K. Boger, Th. Fauster, and M. Weinelt, New J. Phys. 7, 110 (2005).

${ }^{21}$ See Supplemental Material at http://link.aps.org/supplemental/ 10.1103/PhysRevB.86.205403 for details of the photoemission processes and the experimental setup.

${ }^{22}$ D. Rieger, R. D. Schnell, W. Steinmann, and V. Saile, Nucl. Instr. Methods 208, 777 (1983).

${ }^{23}$ K. A. Kokh, B. G. Nenashev, A. E. Kokh, and G. Y. Shvedenkov, J. Cryst. Growth 275, e2129 (2005).

${ }^{24}$ G. Kresse and J. Hafner, Phys. Rev. B 48, 13115 (1993).

${ }^{25}$ G. Kresse and J. Furthmüller, Comput. Mater. Sci. 6, 15 (1996).

${ }^{26}$ P. E. Blöchl, Phys. Rev. B 50, 17953 (1994).

${ }^{27}$ G. Kresse and D. Joubert, Phys. Rev. B 59, 1758 (1999).

${ }^{28}$ J. P. Perdew, K. Burke, and M. Ernzerhof, Phys. Rev. Lett. 77, 3865 (1996).

${ }^{29}$ D. M. Ceperley and B. J. Alder, Phys. Rev. Lett. 45, 566 (1980).

${ }^{30}$ D. D. Koelling and B. N. Harmon, J. Phys. C 10, 3107 (1977).

${ }^{31} \mathrm{http}: / /$ www.flapw.de

${ }^{32}$ C. Li, A. J. Freeman, H. J. F. Jansen, and C. L. Fu, Phys. Rev. B 42, 5433 (1990).

${ }^{33}$ See Ref. 21 for a detailed comparison between the various calculations and experimental results.

${ }^{34}$ J. Klimeš, D. R. Bowler, and A. Michaelides, Phys. Rev. B 83, 195131 (2011).

${ }^{35}$ M. Neupane, S.-Y. Xu, L. A. Wray, A. Petersen, R. Shankar, N. Alidoust, C. Liu, A. Fedorov, H. Ji, J. M. Allred, Y. S. Hor, T.-R. Chang, H.-T. Jeng, H. Lin, A. Bansil, R. J. Cava, and M. Z. Hasan, Phys. Rev. B 85, 235406 (2012).

${ }^{36}$ K. Miyamoto, A. Kimura, T. Okuda, H. Miyahara, K. Kuroda, H. Namatame, M. Taniguchi, S. V. Eremeev, T. V. Menshchikova, E. V. Chulkov, K. A. Kokh, and O. E. Tereshchenko, Phys. Rev. Lett. 109, 166802 (2012).

${ }^{37}$ See Ref. 21 for momentum distribution curves for $\mathrm{Bi}_{2} \mathrm{Se}_{3}$. 
${ }^{38}$ Z. Wang, X.-L. Qi, and S.-C. Zhang, New J. Phys. 12, 065007 (2010).

${ }^{39}$ L. Fu and C. L. Kane, Phys. Rev. B 74, 195312 (2006).

${ }^{40}$ T. Fukui and Y. Hatsugai, J. Phys. Soc. Jpn. 76, 053702 (2007).

${ }^{41}$ R. W. G. Wyckoff, Crystal Structures, Vol. 2. (John Wiley and Sons, New York, 1964).

${ }^{42}$ S. Nakajima, J. Phys. Chem. Solids 24, 479 (1963).

${ }^{43}$ B. M. Goltsman, B. A. Kudinov, and I. A. Smirnov, Thermoelectric Semiconductor Materials Based on $\mathrm{Bi}_{2} \mathrm{Te}_{3}$, (Army Foreign Science \& Technology Center, Charlottesville, VA, 1973), Report FSTCHT-23-1782-73.
${ }^{44}$ S. R. Park, J. Han, C. Kim, Y. Y. Koh, C. Kim, H. Lee, H. J. Choi, J. H. Han, K. D. Lee, N. J. Hur, M. Arita, K. Shimada, H. Namatame, and M. Taniguchi, Phys. Rev. Lett. 108, 046805 (2012).

${ }^{45}$ W. Jung, Y. Kim, B. Kim, Y. Koh, C. Kim, M. Matsunami, S.-i. Kimura, M. Arita, K. Shimada, J. H. Han, J. Kim, B. Cho, and C. Kim, Phys. Rev. B 84, 245435 (2011).

${ }^{46}$ M. R. Scholz, J. Sánchez-Barriga, D. Marchenko, A. Varykhalov, A. Volykhov, L. V. Yashina, and O. Rader, arXiv:1108.1053.

${ }^{47}$ H. Mirhosseini and J. Henk, Phys. Rev. Lett. 109, 036803 (2012).

${ }^{48}$ J. W. McIver, D. Hsieh, H. Steinberg, P. Jarillo-Herrero, and N. Gedik, Nature Nanotechnol. 7, 96 (2012). 